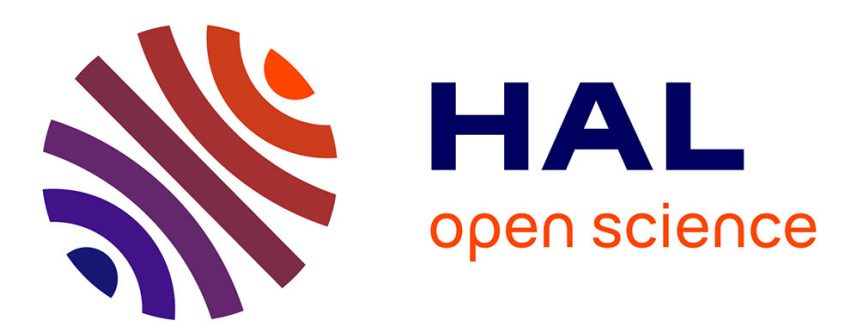

\title{
Characterization of rigid porous medium via ultrasonic reflected waves at oblique incidence
}

Mustapha Sadouki, Amine Berbiche, Mohamed Fellah, Z.E.A Fellah, Claude Depollier

\section{- To cite this version:}

Mustapha Sadouki, Amine Berbiche, Mohamed Fellah, Z.E.A Fellah, Claude Depollier. Characterization of rigid porous medium via ultrasonic reflected waves at oblique incidence. 170th Meeting of the Acoustical Society of America, Nov 2015, Jacksonville, FL, United States. hal-01309356

\section{HAL Id: hal-01309356 https://hal.science/hal-01309356}

Submitted on 2 May 2016

HAL is a multi-disciplinary open access archive for the deposit and dissemination of scientific research documents, whether they are published or not. The documents may come from teaching and research institutions in France or abroad, or from public or private research centers.
L'archive ouverte pluridisciplinaire HAL, est destinée au dépôt et à la diffusion de documents scientifiques de niveau recherche, publiés ou non, émanant des établissements d'enseignement et de recherche français ou étrangers, des laboratoires publics ou privés. 


\section{Proceedings of Meetings on Acoustics \\ Characterization of rigid porous medium via ultrasonic reflected waves at oblique incidence \\ --Manuscript Draft--}

\begin{tabular}{|c|c|}
\hline \multicolumn{2}{|l|}{ Manuscript Number: } \\
\hline Article Type: & ASA Meeting Paper \\
\hline Corresponding Author: & $\begin{array}{l}\text { Mustapha Sadouki } \\
\text { Université djillali bounaama } \\
\text { Khemis-Miliana, Ain Defla ALGERIA }\end{array}$ \\
\hline \multirow[t]{4}{*}{ Order of Authors: } & Mustapha Sadouki \\
\hline & Amine Berbiche \\
\hline & Mohamed Fellah \\
\hline & Zine.E.A Fellah \\
\hline Section/Category: & Physical Acoustics \\
\hline
\end{tabular}




\title{
(s). Proceedings of Meetings on Acoustics
}

Volume 25

http://acousticalsociety.org/

\section{Meeting of the Acoustical Society of America}

Jacksonville, Florida

2-6 November 2015

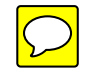

General Topics in Physical Acoustics II: Paper 5aPA

\section{Characterization of rigid porous medium via ultrasonic reflected waves at oblique incidence}

\author{
Mustapha. Sadouki \\ Fac. des Sciences et de la Technologie, Université de Khemis Miliana, BP 44225, Ain Defla, Algérie \\ mustapha.sadouki@univ-dbkm.dz
}

\section{Amine. Berbiche}

Lab. de Physique Théorique, Faculté de Physique, USTHB, BP 32, 16111 El Alia, Bab Ezzouar, Algérie amine.berbiche@yahoo.fr

\section{Mohamed. Fellah}

Lab. de Physique Théorique, Faculté de Physique, USTHB, BP 32, 16111 El Alia, Bab Ezzouar, Algérie mfellah@usthb.dz

\section{Zine. E. A. Fellah}

LMA UPR7051 CNRS Aix-Marseille Univ, Centrale Marseille, 31 chemin Joseph Aiguier, F-13402 Marseille Cedex, 20, France fellah@lma.cnrs-mrs.fr

\section{Claude. Depollier}

LUNAM Universite du Maine, UMR CNRS 6613 Laboratoire d'Acoustique de l'Université du Maine UFR STS Avenue O. Messiaen 72085 Le Mans CEDEX 09 France claude.depollier@ univ-lemans.fr

In this paper, an enhanced method is proposed for measuring porosity, tortuosity, viscous and thermal characteristic length of porous materials having a rigid frame via reflected ultrasonic waves at oblique incidence using the equivalent fluid model. The advantage of the proposed method is that the four parameters are determined simultaneously just using reflected experimental waves for a porous material saturated by air. The inverse problem is solved based on the least-square numerical method using experimental reflected waves in time domain. Tests are performed using industrial plastic foams. Experimental and numerical validation results of this method are presented.

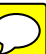




\section{Introduction}

In this work, we present a reflectivity method for measuring the physical parameters describing the propagation of ultrasonic pulses in air-saturated porous materials. This method is based on a temporal model of direct and inverse scattering problems for the propagation of transient ultrasonic waves in a homogeneous isotropic slab of porous material with a rigid frame, The principle of this method is to measure the wave reflected at oblique incidence by the slab of porous material, and to solve the inverse problem in order to estimate the physical parameters (porosity, tortuosity, viscous and thermal characteristic lengths). The advantage of this method over classic ultrasonic methods is that all these parameters can be determined simultaneously using experimental reflected data.

\section{Model}

In air-saturated porous media, the structure is generally motionless and the waves propagate only in the fluid. This case is described by the equivalent fluid model which fluid-structure interactions are taken into account in two frequency response factors: dynamic tortuosity of the medium $\alpha(\omega)$ given by Johnson et al. ${ }^{1}$ and dynamic compressibility of the air in the porous material $\beta(\omega)$ given by Allard ${ }^{2}$. The ultrasonic regime corresponds to the range of frequencies such that viscous skin thickness $\boldsymbol{\delta}=\sqrt{\mathbf{2 \eta} / \boldsymbol{\omega} \rho_{f}}$ is much small than the radius of the pores $r, \boldsymbol{\delta} / \boldsymbol{r} \ll \mathbf{1}$. In the high frequency the expressions of the responses factors $\alpha(\omega)$ and $\beta(\omega)$ are given by ${ }^{1,2}$ :

$$
\begin{gathered}
\alpha(\omega)=\alpha_{\infty}\left(1+\frac{2}{\Lambda}\left(\frac{\eta}{j \omega \rho_{f}}\right)^{\frac{1}{2}}\right), \\
\beta(\omega)=1+\frac{2(\gamma-1)}{\Lambda^{\prime}}\left(\frac{\eta}{P_{r} \rho_{f}}\right)^{1 / 2}\left(\frac{1}{j \omega}\right)^{1 / 2},
\end{gathered}
$$

In these equations, $\omega$ is the pulsation, $\operatorname{Pr}$ is the Prandtl number, $\eta$ and $\rho_{\mathrm{f}}$ are, respectively, the fluid viscosity and the fluid density, and $\gamma$ is the adiabatic constant. The relevant physical parameters of the model are the tortuosity of the medium $\alpha_{\infty}$ initially introduced by Zwikker and Kosten ${ }^{10}$, the viscous and the thermal characteristic lengths $\Lambda$ and $\Lambda^{\prime}$ introduced by Johnson et al. ${ }^{1}$ and Allard ${ }^{2}$.

\section{Direct problem}

The direct scattering problem is that of determining the scattered field as well as the internal field that arises when a known incident field impinges on the porous material with known physical properties. The geometry of the problem is shown in Fig.1.

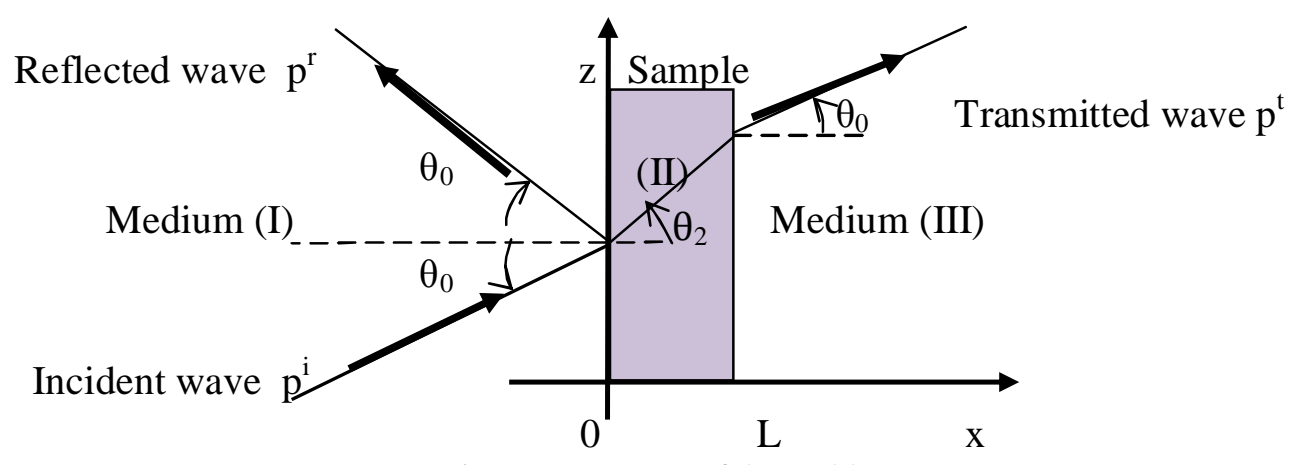

Fig.1. - Geometry of the problem. 
A homogeneous porous material occupies the region $0<x<L$. This medium is assumed to be isotropic and to have a rigid frame. A short sound pulse impinges at oblique incidence on the medium from the left, it gives rise to an acoustic pressure field $p(x, z, t)$ and an acoustic velocity field $v(x, z, t)$ within the material, which satisfying the Euler equation and the constitutive equation (along the $\mathrm{x}$ axis):

$$
\rho \alpha(\omega) j \omega v=\nabla p, \quad \frac{\beta(\omega)}{K_{a}} j \omega p=\nabla \cdot v
$$

where $j^{2}=-1, \rho$ is the suturing fluid density, and $\mathrm{K}_{\mathrm{a}}$ is the compressibility modulus of the fluid. The expression of the acoustic fields and velocity in each medium $(1, . ., 3)$ are given by The expressions of a pressure wave and velocity arriving at oblique incidence in each medium $(1,2,3)$ are given by:

$$
\begin{gathered}
P_{1}(x, z, \omega)=\left(e^{-j k_{1} x \cos \theta_{0}}+R(\omega) e^{j k_{1} x \cos \theta_{0}}\right) e^{-j\left(k_{1} z \sin \theta_{0}-\omega t\right)}, \\
V_{1}(x, z, \omega)=\frac{\cos \theta_{0}}{Z_{1}}\left(e^{-j k_{1} x \cos \theta_{0}}-R(\omega) e^{j k_{1} x \cos \theta_{0}}\right) e^{-j\left(k_{1} z \sin \theta_{0}-\omega t\right)}, \\
P_{2}(x, z, \omega)=\left(A(\omega) e^{-j k_{2} x \cos \theta_{2}}+B(\omega) e^{j k_{2} x \cos \theta_{1}}\right) e^{-j\left(k_{2} z \sin \theta_{2}-\omega t\right)}, \\
V_{2}(x, z, \omega)=\frac{\cos \theta_{2}}{Z_{2}}\left(A(\omega) e^{-j k_{2} x \cos \theta_{2}}-B(\omega) e^{j k_{2} x \cos \theta_{2}}\right) e^{-j\left(k_{2} z \sin \theta_{2}-\omega t\right)}, \\
P_{3}(x, \omega)=T(\omega) e^{-j k_{3}(x-L) \cos \theta_{0}} e^{-j\left(k_{3} z \sin \theta_{0}-\omega t\right)}, \\
V_{3}(x, z, \omega)=\frac{\cos \theta_{0}}{Z_{3}} T(\omega) e^{-j k_{3}(x-L) \cos \theta_{0}} e^{-j\left(k_{3} z \sin \theta_{0}-\omega t\right)},
\end{gathered}
$$

Where $\theta_{0}$ is the incident angle, $\theta_{2}$ is the refracted angle in the medium (II), $R(\omega), T(\omega)$ are the reflected and transmitted coefficients, $A(\omega)$ and $B(\omega)$ are function of pulsation for determining, $z_{i}(\omega)=\sqrt{\rho k_{a} \alpha_{i}(\omega) / \beta_{i}(\omega)}$ and $k_{i}(\omega)=\omega \sqrt{\rho \alpha_{i}(\omega) \beta_{i}(\omega) / k_{a}}$ are the characteristic impedance and the wave number, respectively, of the acoustic wave in each medium $(i=1,2,3) . k_{a}$ is the compressibility module of the fluid. To derive the reflection coefficient in frequency domain, it is assumed that the pressure field and velocity are continuous at the boundary of the medium

$$
\begin{aligned}
& p_{1}\left(0^{-}, \omega\right)=p_{2}\left(0^{+}, \omega\right), \quad p_{2}\left(L^{-}, \omega\right)=p_{3}\left(L^{+}, \omega\right), \\
& v_{1}\left(0^{-}, \omega\right)=\phi v_{2}\left(0^{+}, \omega\right), \quad \phi v_{2}\left(L^{-}, \omega\right)=v_{3}\left(L^{+}, \omega\right)
\end{aligned}
$$

where $\phi$ is the porosity of the medium and the \pm superscript denotes the limit from right and left, respectively. Using boundary and initial condition (10), reflection coefficient can be derived:

$$
R(\omega)=\frac{\left(1-E^{2}(\omega)\right) \sinh (j k(\omega) L)}{2 E(\omega) \cosh (j k(\omega) L)+\left(1+E^{2}(\omega)\right) \sinh (j k(\omega) L)}
$$

with,

$$
E(\omega)=\phi \frac{\cos \theta_{2}}{\cos \theta_{0}} \sqrt{\frac{\beta(\omega)}{\alpha(\omega)}}, k(\omega)=\omega \sqrt{\frac{\rho \alpha(\omega) \beta(\omega)}{K_{a}}} \cos \theta_{2} \text { and } \cos \theta_{2}=\sqrt{1-\frac{\sin \theta_{0}{ }^{2}}{\alpha(\omega) \beta(\omega)}}
$$

The incident and scattered fields are related by the reflection operators by:

$$
p^{r}(x, t)=\int_{0}^{t} \tilde{R}(\tau) p^{i}\left(t-\tau+\frac{x}{c_{0}}\right) d \tau=\tilde{R}(t) * p^{i}(t) * \delta\left(t+\frac{x \cos \theta_{0}}{c_{0}}\right)
$$


In Eq. (15) * denotes the convolution operation, $\tilde{R}(t)$ is the reflection kernel, its temporal expression is calculated numerically by taking the inverse Fourier transform of Eq.(11), $p^{i}(t)$ is the incident field and $\delta$ is the Delta function.

\section{Inverse problem}

A basic inverse problem associated with the slab may be stated as follows: by measuring reflected signal by the slab, find the values of the medium's parameters. So, our objective is to find values of $\phi$, $\alpha_{\infty}, \Lambda$ and $\Lambda^{\prime}$ knowing the reflection coefficient for different values of the incidence angle $\theta_{0}$. The inverse problem is solved numerical by minimize the cost function $U\left(\phi, \alpha_{\infty}, \Lambda, \Lambda^{\prime}\right)$ defined by $U\left(\phi, \alpha_{\infty}, \Lambda, \Lambda^{\prime}\right)=\sum_{i=1}^{i=N}\left(p^{r}\left(x, t_{i}\right)-p_{\text {exp }}^{r}\left(x, t_{i}\right)\right)^{\wedge} 2$ where $p_{\text {exp }}^{r}\left(x, t_{i}\right)$ is the discrete set of values of the experimental reflected signal and $p^{r}\left(x, t_{i}\right)$ is the discrete set of values of the simulated reflected signal. The parameters of the first investigated plastic foam M1 are: thickness $4.1 \mathrm{~cm}$, The value of the porosity given by the porosimeter ${ }^{9}$ is $\phi=0.9 \pm 0.05$, and the values of the tortuosity $\alpha_{\infty}$, viscous and thermal characteristic length $\Lambda, \Lambda^{\prime}$ given by classical method ${ }^{3-9}$ are $\alpha_{\infty}=1.06 \pm 0.08, \Lambda=230 \pm 50 \mu \mathrm{m}$ and the ratio between $\Lambda$ and $\Lambda^{\prime}$ is fixed at $3\left(\Lambda^{\prime} / \Lambda=3\right)$. Fig.2 shows the incident signal generated by the transducer (solid line) and the reflected signal by the plastic foam M1 (dashed line) and their spectra for an incidence angle $\theta=17^{\circ}$. The optimized values of these parameters are given in table 1 . We present in Fig. 3 the variation in the minimization function $U$ given in with porosity, tortuosity, viscous and thermal characteristic length, respectively. In Fig. 4 we show a comparison of an experimental reflected signal and a simulated signal obtained by optimization from the inverse problem. The correlation of theoretical prediction and experimental data is good.
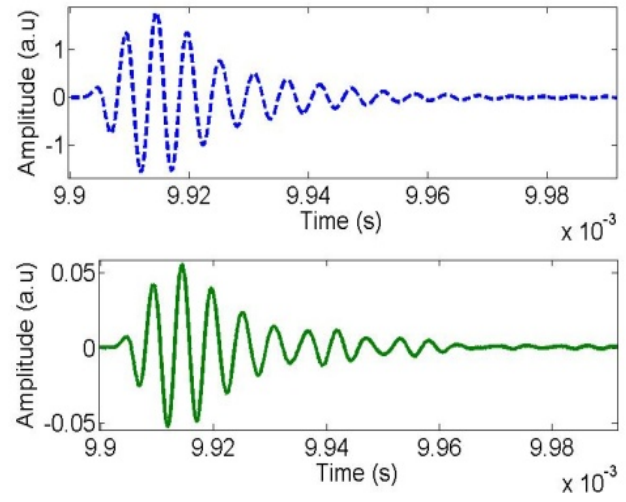
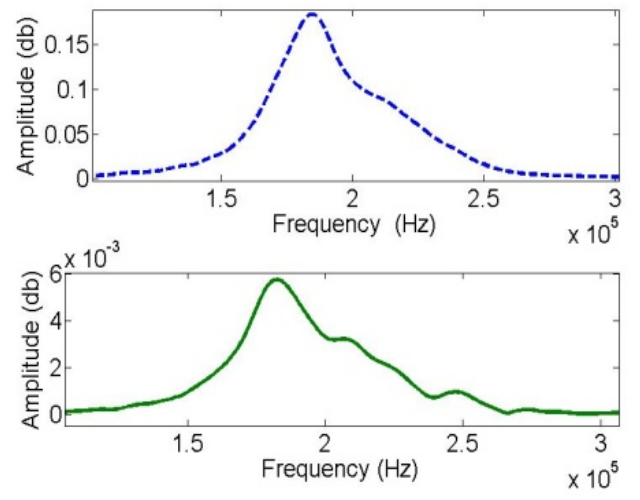

Fig.2. Experimental incident signal (dashed line) and experimental reflected signal (solid line) at left and their spectrum at right $\left(\theta=17^{\circ}\right)$.

Table1. Values of the parameters obtained by optimization from the inverse problem.

\begin{tabular}{clllll} 
Angle $\left(^{\circ}\right)$ & $\phi$ & $\alpha_{\infty}$ & $\Lambda(\mu \mathrm{m})$ & $\Lambda^{\prime} / \Lambda$ & $\Lambda^{\prime}(\mu \mathrm{m})$ \\
\hline $\mathbf{0}$ & 0.86 & 1.06 & 257.14 & 1.6 & 404.08 \\
$\mathbf{1 7}$ & 0.86 & 1.06 & 257.14 & 1.6 & 404.08 \\
$\mathbf{2 3}$ & 0.86 & 1.05 & 257.14 & 1.6 & 404.08 \\
$\mathbf{3 5}$ & 0.83 & 1.05 & 257.14 & 1.6 & 404.08 \\
\hline
\end{tabular}



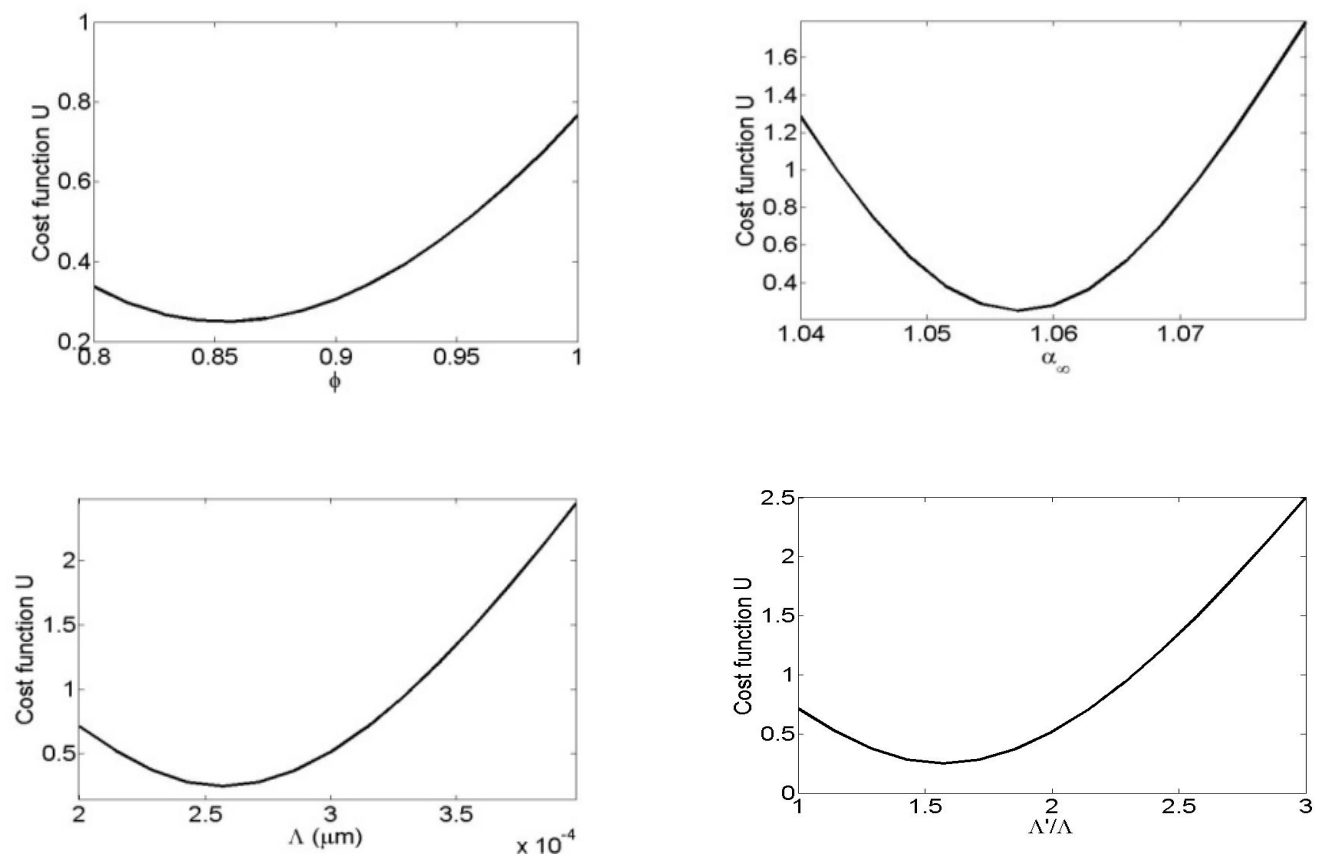

Fig.3.- Variation of the minimization function $U$ with porosity, tortuosity, viscous characteristic length and the ratio $\Lambda^{\prime} / \Lambda$ at $\theta=17^{\circ}$

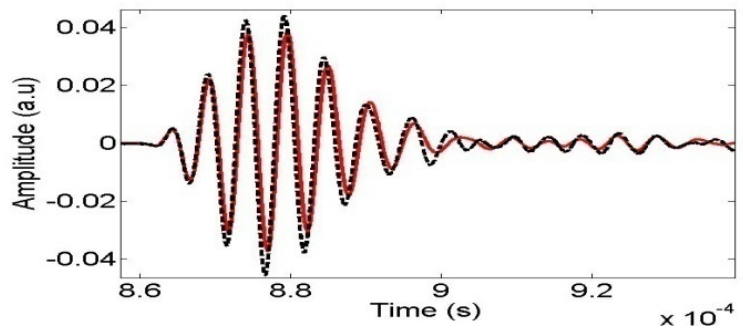

Fig.5- Comparison between experimental reflected signal (dashed line) And simulated reflected signal (solid line) of the sample M1.

\section{Conclusion}

In this paper, a method for measuring transport parameters in porous materials simultaneously, using measurements of ultrasonic waves reflected at incidence oblique has been proposed. This method is based on a temporal model of direct and inverse problems affecting the propagation of transient waves in a homogeneous isotropic slab of porous material with a rigid frame. The advantage of the proposed method is that all the parameters can be determined by reflected waves at oblique incidence. An experimental validation of the theoretical expression of the reflection coefficient illustrates the high level of correspondence between numerical and experimental results and show that this model is well suited for the characterization of porous media via acoustic wave propagation. This method is an alternative to the usual method involving the use of transmitted waves. 


\section{References}

1 A. D. L. Johnson, J. Koplik, and R. Dashen, 1987." Theory of dynamic permeability and tortuosity in fluid-saturated porous media " J. Fluid Mech. 176, 379

2 J. F. Allard 1993., "Propagation of Sound in Porous Media" (Chapman and Hall), London,

3 ZEA Fellah, M Sadouki, M Fellah, F. G Mitri, E Ogam, C Depollier. 2013” Simultaneous determination of porosity, tortuosity, viscous and thermal characteristic lengths of rigid porous materials" J. Appl. Phys, 114, 204902;

4 ZEA. Fellah, S. Berger and W.Lauriks, C. Depollier, C. Aristegui and J.Y. Chapelon 2003 "Measuring the porosity and the tortuosity of porous materials via reflected waves at oblique incidence" J. Acoust. Soc. Am. 113(5), 2424-2433.

5 Z. E. A. Fellah, C. Depollier, and M. Fellah, 2002 "Application of Fractional Calculus to the Sound Waves Propagation in Rigid Porous Materials: Validation Via Ultrasonic Measurements" Acta Acust. (Beijing) 88, 34.

6 P. Leclaire, L. Kelders, W. Lauriks, N. R. Brown, M. Melon, and B. Castagne 1996. "Determination of the viscous and thermal characteristic lenghts of plastic foams by ultrasonic measurements in helium and air", J. Appl. Phys. 80, 2009-2012

7 A. Moussatov, C. Ayrault, and B. Castagnéde 2001., "Porous material characterization-ultrasonic method for estimation of tortuosity and characteristic length using a barometric chamber" Ultrasonics 39, 195-202

8 N. Brown, M. Melon, V. Montembault, B. Castagne`de, W. Lauriks, and P. Leclaire 1996 , "Evaluation of viscous characteristic length of air-saturated porous materials from the ultrasonic dispersion curve," C. R. Acad. Sci. Paris, 322, 121-127.

9 Y. Champoux, M. R. Stinson, and G. A. Daigle, 1991 "Air-based system for the measurement of porosity"' J. Acoust. Soc. Am. 89, 910-916

10 C. Zwikker and C. W. Kosten, 1949. "Sound Absorbing Materials “ Elsevier, New York, 